\title{
Artificial Intelligence in Education
}

\author{
Matthew N. O. Sadiku1*, Tolulope J. Ashaolu², and Abayomi Ajayi-Majebi, ${ }^{3}$ and Sarhan M. Musa1
}

\author{
${ }^{1}$ Roy G. Perry College of Engineering, Prairie View A\&M University, Prairie View, TX, USA \\ ${ }^{2}$ College of Food Science, Southwest University, Tiansheng Road Beibei District, Chongqing, 400715, P.R. China \\ ${ }^{3}$ Department of Manufacturing Engineering, Central State University, P.0. Box 1004, Wilberforce, OH, USA \\ E-mail: sadiku@ieee.org; ashaolut@gmail.com; ajayi-majebi@centralstate.edu; smmusa@pvamu.edu
}

*corresponding author details: Professor Matthew N. O. Sadiku; sadiku@ieee.org

\begin{abstract}
Artificial intelligence refers to the ability of a computer system to perform human tasks (such as thinking and learning) that usually can only be accomplished using human intelligence. AI technology in education is allowing a degree of flexibility and customization that was never before possible. It is revolutionalizing schools and classrooms, making educator's job a lot easier. It is poised to revolutionize education. This paper provides various applications of $\mathrm{AI}$ in education.
\end{abstract}

Keywords: artificial intelligence; education; learning; teaching

\section{INTRODUCTION}

Education is a process where teachers give systematic instructions, while students receive them. It is a major determining factor for an individual's success in life.

Education seems to be fixed in terms of time, place, and prescribed activities. Learning takes place continuously, especially for younger people. Traditional education systems are known to be inflexible but is now changing to adapt to the technological advancements of today's world. One key technology that is poised to transform education is artificial intelligence (AI). The implementation of AI has several benefits for students and teachers alike [1].

$\mathrm{AI}$ is a general-purpose technology that can perform tasks that previously required human beings. It can be regarded as the simulation of human mental capacity by the machines. It is a domain of research with many subdisciplines, domains of expertise, and developmental dynamics. As shown in Figure 1, AI is rapidly transforming many industries such as healthcare, transportation, business, retail, construction, food industry, manufacturing, and education [2].

Apple's Siri voice assistant, Amazon's shopping recommendations, Uber ride sharing, and Google translate are common examples of how AI has invaded our daily lives [3]. AI It has impacted almost all sectors of human life and provided a lot of benefits to various fields, including education. It is having a positive impact on educational applications. The field of education is a place where artificial intelligence is poised to make big changes. Artificial Intelligence in Education (AIEd) has become a field of scientific research for more than 30 years. AIEd is mainly concerned with the development of "computers which perform cognitive tasks, usually associated with human minds' [4].

\section{OVERVIEW ON ARTIFICIAL INTELLIGENCE}

Artificial intelligence (AI) is one of the most important global issues of the 21 st century.
The modern field of artificial intelligence came into existence in 1956 when the term "artificial intelligence" (AI) was coined by John McCarthy. AI is the branch of computer science that deals with designing intelligent computer systems that mimic human intelligence (e.g. visual perception, speech recognition, decision-making, and language translation). The ability of machines to process natural language, to learn, to plan makes it possible for new tasks to be performed by intelligent systems. The main purpose of $\mathrm{AI}$ is to mimic the cognitive function of human beings and perform activities that would typically be performed by a human being. Without being taught by humans, machines use their own experience to solve a problem. Although the original vision for artificial intelligence was to simulate human intelligence, research effort has gradually shifted to autonomous systems that compete with people.

AI is stand-alone independent electronic entity that functions much like human expert. Today, AI is integrated into our daily lives in several forms, such as personal assistants, automated mass transportation, aviation, computer gaming, facial recognition at passport control, voice recognition on virtual assistants, driverless cars, companion robots, etc. AI technologies are performing better and better at analyzing data $[5,6]$.

An importantfeature of AI technology is that is can be added to existing technologies. AI has benefited many areas such chemistry and medicine, where routine diagnoses can be initiated by AI-aided computers. It embraces a wide range of disciplines such as computer science, engineering, chemistry, biology, physics, astronomy, neuroscience, and social sciences.

$\mathrm{AI}$ is not a single technology but a range of computational models and algorithms. The major disciplines in AI include expert systems, fuzzy logic, and artificial neural networks (ANNs), machine learning, deep learning, natural language processing, computer vision, and robotics. These computerbased tools or technologies that have been used to achieve AI's goals are illustrated in Figure 2 [7] and explained as follows [8,9]: 
- Expert Systems: An expert system (ES) (or knowledgebased system) essentially embodies the knowledge and reasoning of human experts. An ES enables computers to make decisions by interpreting data and selecting between alternatives just as a human expert would do. It uses a technique known as rule-based inference in which rules are used to process data. The main business application of AI is in expert systems, which assist human experts in solving difficult problems.

- Neural Networks: These computer programs identify objects or recognize patterns after having been trained Artificial neural networks (ANNs) are parallel distributed systems consisting of processing units (neurons) that calculate some mathematical functions. The ANN model represents nonlinear relationships which are directly learned from the data being modeled.

- Computer Vision: Computer vision comprehends methods and techniques through which artificial vision systems can be constructed. Essentially, a computer vision system is composed of image acquisition and image processing. Computer vision. systems are already widely employed in different segments of agricultural production.

- Natural Language Processors: For AI to be useful to us humans, it needs to be able to communicate with us in our language. Computer programs can translate or interpret language as it is spoken by normal people. NLP techniques extract information from unstructured data such as clinical notes to supplement and enrich structured medical data. NLP includes applications such as speech recognition, text analysis, translation and other goals related to language. There are two basic approaches to NLP: statistical and semantic [11]. NLP allows for intelligent search engines, helpful chatbots, and accessibility for people who are visually impaired.

- Robots: These are computer-based programmable machines that have physical manipulators and sensors. Sensors can monitor temperature, humidity, pressure, time, record data, and make critical decisions in some cases. Robots have moved from science fiction to your local hospital. In jobs with repetitive and monotonous functions they might even completely replace humans. Robotics and autonomous systems are regarded as the fourth industrial revolution.

- Fuzzy Logic: This involves reasoning based on imprecise or incomplete information in terms of a range of values rather than point estimates. Fuzzy logic deals with uncertainty in knowledge that simulates human reasoning in incomplete or fuzzy data. The fuzzy model is robust to parameter changes and tolerant to impression.

- Machine Learning: Algorithms make predictions and interpret data and "learn" without static program instructions. ML is a statistical technique for fitting models to data and training models with data. ML extracts features from input data by constructing analytical data algorithms and examines the features to create predictive models. The most common ML algorithms are supervised learning, unsupervised learning, reinforcement learning, and deep learning. ML algorithms are a good fit for anti-malware solutions because machine learning is well suited to solve "fuzzy" problems. AI has developed in waves, with each wave bringing a capability that was thought impossible in the previous waves. Today, artificial intelligence is narrow and mainly based on machine learning.
- Deep Learning: A subset of machine learning built on a deep hierarchy of layers, with each layer solving different pieces of a complex problem. It aims at increasing the capacity of supervised and unsupervised learning algorithms for solving complex real-world problems by adding multiple processing [12]. The relationship between artificial intelligence, machine learning, and deep learning is shown in Figure 3 [13]

- Data Mining: This deals with the discovery of hidden patterns and new knowledge from large databases. Data mining exhibits a variety of algorithmic tools such as statistics, regression models, neural networks, fuzzy sets, and evolutionary models.

Each AI tool has its own advantages. Using a combination of these models, rather than a single model, is recommended. AI technologies are drastically influencing the retail industry and customer experience. Some types of artificial intelligence are predominantin business, while others are not. AI systems are designed to make decisions using real-time data. They have the ability to learn and adapt as they make decisions.

\section{APPLICATIONS OF AI IN EDUCATION}

Figure 4 shows a typical example on how $\mathrm{AI}$ is changing the education industry [14].

There are several AI applications for education. Popular areas to incorporate AI technologies to facilitate students' learning include engineering education, higher education, mathematics education, language education, surgical education, robotics education, computer science education, STEM education, medical education, musical education, and science education [15]. Companies using $\mathrm{AI}$ in education to enhance the classroom include Nuance, Knewton, Cognii, Querium, Century Tech, KidSense, Carnegie Learning, Kidaptive, Blippar, Thinkster Math, Volley, and Quizlet. These companies are merging the organic and the artificial by applying AI tools to innovate how people are educated [16].

Figure 5 compares traditional education with AI-driven education [17]. An application is the use of expert systems to assist with educational diagnosis and assessment. Other applications include the following.

- In the Classroom: While AI can never replace human teachers, it can play a great role in the classroom. AI can allow teachers to hand off all assignments to an AI for grading so the teachers can spend more time with students. Despite the cost and need for Internet, AI is useful for tutoring. Since teachers cannot be available for students all the time, tutors are needed. Working with an AI tutor can help students with social or academic anxiety get the help [18].

- Personalized Education: Traditional education is not flexible. AI will enable personalized or tailored education. AI can provide a level of differentiation that customizes learning specifically to an individual student. Artificial intelligence helps build a personalized study schedule for each learner, thereby tailoring studies according to student's specific needs. This opens up new ways of interacting for students with learning disabilities. Personalized education increases efficiency, improves accessibility, and scales the processes [19].

- Administration: AI can simplify administrative tasks. It can automate the expedition of administrative duties for teachers and academic institutions. Technology can be used to automate the grading tasks where multiple tests are involved. This means that professors would have 
more time with their students rather than spending long hours grading them.

- Universal Access to Global Classrooms: AI can help to eliminate boundaries, thereby facilitating the learning of any course from anywhere, anytime across the globe. Al tools can help make global classrooms available to all including those who speak different languages. There will be a wider range of courses available online and with the help of AI, students will be learning from wherever they are [20].

- Medical Education: Essentially, medicine serves common human needs and promotes patient well-being. AI is critical for the future development of healthcare. The rate at which emerging health AI technologies are developing, being introduced into clinical practice, and being used by patients requires that healthcare professionals are well equipped. Physicians must work with patients to make the implementation of $\mathrm{AI}$ technologies transparent and accountable [21]. AI can be used to check the effectiveness of the medical program and satisfaction of the medical students.

- Marketing Education: Artificial intelligence is transforming marketing profession. It has several applications in marketing. These include sales forecasting, website experience personalization, speech recognition, content creation, chatbots, etc. For example, chatbot is regarded as one of the most promising $\mathrm{Al}$ applications. The virtual assistant is used in online marketing. Learning to comprehend and use AI can be beneficial to students. Marketing students believe that they will work AI tools during their careers [22].

Other applications include personalized guidance, supports, feedback, assessment tools, remediation, virtual assistants for students, mobile games, intelligent tutoring systems, educational robots, smart education, engineering education, and design education.

\section{BENEFITS}

AI tools are gradually changing the landscape of education. They can help make global classrooms available to all. Today, AI applications in education (AIEd) are widely used by learners and educators. Although AI provides many benefits for students and teachers, it cannot replace teachers. The benefits of AI for students, teachers, and school include [23,24]:

- Education at any time: $\mathrm{AI}$ is impacting on how children can learn and from where. AI-based applications allow students to study whenever they are free time and receive feedback from teachers in a real-time mode.

- Virtual mentors: AI-based platforms offer virtual mentors to track the students' progress. AI holds promise as a tool to monitor student performance.

- Better engagement: Modern technologies like VR and gamification help involve students in the education process, making it more interactive and personalized.

- Opportunity to find a good teacher: Educational platforms have a lot of teachers, so the student has an opportunity to communicate with specialists from other countries.

- Personalized learning: Already discussed.
- Teacher's aid: AI can be a great ally to a teacher. AI can help an educator reduce the burden of administrative duties such as marking exams, grading students' assignments, planning, etc. and save a lot of time.

- Teaching the teacher: Artificial intelligence makes comprehensive information available to teachers any time of day.

- Connecting everyone: AI tools can help make global classrooms available to all, fostering greater cooperation, communication, and collaboration among schools and nations.

- Cost reduction: AI can accelerate and reduce the cost of learning.

- Improves Efficiency: AI reduces the burden of repetitive tasks teachers and schools have to deal with daily. For example, students can use the AI-powered tools to learn word pronunciations, meaning, and proper usage. From classroom interactions, coursework learning, and admin processes, AI makes things better and efficient.

\section{CHALLENGES}

Although the possibilities of AI are exciting, a number of challenges prevent the full realization of AI in various educational institutions. Those challenges include:

- Limited capability: The capabilities of Artificial Intelligence in education are limited.

- Unanswered questions: There are a lot of unanswered questions about AI's role and how it will be managed in higher education.

- Trust: Parents and school administrators may find it difficult to trust AI technologies used to influence or make decisions about student learning.

- Ethical dilemma: This is a situation to which the human being is confronted when he opposes, on the one hand, morality or conscience and on the other, life. Another ethical dilemma faced by today's society is the "humanism" of the human being.

\section{CONCLUSION}

AI is impacting education in various ways and this is expected to continue. AI has produced educational tools which have attracted attention for their potential to improve education quality and enhance teaching and learning methods. As AI educational solutions matures, AI will be able to help fill needs gaps. There is no denying the place of AI in modern teaching and learning. Although the critical presence of teachers is irreplaceable, there will be many changes to a teacher's responsibilities. In the coming decades, AI will transform education.

Since students of today will work in an environment where AI is the reality, it is expedient that our academic institutions expose students to AI technology.

More information on artificial intelligence in the food industry can be found in the books in [25-32] and following related journals:

- Artificial Intelligence Review

- International Journal of Artificial Intelligence in Education

- Computers \& Education: Artificial Intelligence

- International Journal of Learning Analytics and Artificial Intelligence for Education 


\section{REFERENCES}

[1] P. Dadhich, "Impact of artificial intelligence on the current education system,"

September 2020, https://wire19.com/impact-of-artificialintelligence-oneducationsystem/\#:

:text=The $\% 20$ implementation $\% 20$ of $\% 20$ educational $\%$ $20 \mathrm{AI}$,

more $\% 20$ knowledge $\% 20$ in $\% 20$ multiple $\% 20$ subjects.

\&text=The $\% 20$ implementation $\% 20$ of $\% 20 \mathrm{AI} \% 20$ can, well $\% 20$ as $\% 20$ streamline $\% 20$ administrative $\% 20$ tasks.

[2] R. Johnson, "Jobs of the future: Starting a career in artificial intelligence," May 2020,

https://www.bestcolleges.com/blog/future-proofindustries-artificial-intelligence/

[3] "Ten facts about artificial intelligence in teaching and learning,"

https://teachonline.ca/sites/default/files/tools-

trends/downloads/ten_facts_about_artificial_intelligence $0 . p d f$

[4] X. Chen et al., "Application and theory gaps during the rise of artificial intelligence in education," Computers and Education: Artificial Intelligence, vol.1, 2020.

[5] M. N. O. Sadiku, T. J. Ashaolu, and S. M. Musa," Artificial intelligence in medicine: A primer," International Journal of Trend in Research and Development, vol. 6, no. 1, January. -Feb. 2019, pp. 270-272.

[6] Y. Mintz and R. Brodie, "Introduction to artificial intelligence in medicine," Minimally Invasive Therapy \& Allied Technologies, vol. 28, no. 2, 2019, pp. 73-81.

[7] O. Oana, T. Cosmin, and N. C. Valentin, "Artificial intelligence - A new field of computer science which any business should consider," "Ovidius" University Annals, Economic Sciences Series, vol. XVII, no. 1, 2017, pp. 356360.

[8] R. O. Mason, "Ethical issues in artificial intelligence," Encyclopedia of Information Systems, vol 2, 2003, pp. 239258.

[9] A. N. Rames et al., "Artificial intelligence in medicine," Annals of the Royal College of Surgeons of England, vol. 86, 2004, pp. 334-338.

[10] D. I. Patrícioa and R. Rieder, "Computer vision and artificial intelligence in precision agriculture for grain crops: A systematic review," Computers and Electronics in Agriculture, vol. 153, October 2018, pp. 69-81.

[11] M. N. O. Sadiku, Y. Zhou, and S. M. Musa, "Natural language processing in healthcare," International Journal of Advanced Research in Computer Science and Software Engineering, vol. 8, no. 5, May 2018, pp. 39-42.

[12] F. Jiang et al., "Artificial intelligence in healthcare: Past, present and future," Stroke and Vascular Neurology, 2017.

[13] "Quantum computing,"

https://quantumcomputingtech.blogspot.com/2019/05/ ai-machine-learning-venn-diagram.html

[14] https://elearningindustry.com/ai-is-changing-theeducation-industry-5-ways
[15] X. Chen, H. Xie, and G. Hwang, "A multi-perspective study on artificial intelligence in education: grants, conferences, journals, software tools, institutions, and researchers," Computers and Education: Artificial Intelligence, vol.1, 2020.

[16] A. Schroer, "12 Companies using AI in education to enhance the classroom," March 2020,

https://builtin.com/artificial-intelligence/ai-in-education

[17] https://www.educationworld.in/how-ai-ineducation-can-dominate-in-2020/

[18] A. Sears, "The role of artificial intelligence in the classroom," April 2018,

https://elearningindustry.com/artificial-intelligence-inthe-classroom-role

[19] L. Plitnichenko, "5 Main roles of artificial intelligence in education," May 2020,

https://elearningindustry.com/5-main-roles-artificialintelligence-in-education

[20] B. Marr, "How is AI used in education -- Real world examples of today and a peek into the future," July 2018, https://www.forbes.com/sites/bernardmarr/2018/07/2 5/how-is-ai-used-in-education-real-world-examples-oftoday-and-a-peek-into-the-future/?sh=2aea5075586e

[21] V. Rampton, M. Mittelman, and J. Goldhahn, "Implications of artificial intelligence for medical education,"

https://www.thelancet.com/journals/landig/article/PIIS 2589-7500(20)30023-6/fulltext

[22] S. Elhajjar, M. S. Karam, and S. Borna, "Artificial intelligence in marketing education programs," Marketing Education Review, 2020.

[23] V. Kuprenko, "Artificial intelligence in education: Benefits, challenges, and use cases," https://medium.com/towards-artificialintelligence/artificial-intelligence-in-education-benefitschallenges-and-use-cases-db52d8921f7a

[24] "The role of artificial intelligence in the future of education," March 2019,

https://www.getsmarter.com/blog/market-trends/therole-of-artificial-intelligence-in-the-future-of-education/

[25] Artificial Intelligence in Education; Building Technology Rich Learning Contexts That Work, (Int'l Conference on Artificial Intelligence in Education, 2007, Los Angeles, CA). IOS Press, 2007.

[26] U. Kose and D. Koc (eds.), Artificial Intelligence Applications in Distance Education. Information Science Reference, 2015

[27] R. J. Spiro, B. C. Bruce, and W. F. Brewer, Theoretical Issues in Reading Comprehension: Perspectives from Cognitive Psychology, Linguistics, Artificial Intelligence and Education. Taylor \& Francis, 2017.

[28] R. W. Lawler and M. Yazdani, Artificial Intelligence and Education: Learning environments and tutoring systems. Intellect Ltd, 1987.

[29] C. Fadel, W. Holmes, and M. Bialik, Artificial Intelligence in Education: Promises and Implications for Teaching and Learning. Independently published, 2019. 
[30] G. Gauthier and C. Frasson (eds.), Intelligent Tutoring Systems: At the Crossroad of Artificial Intelligence and Education. Intellect Ltd, 1990.

[31] R. M. Cameron, A.I. - 101: A Primer on Using Artificial Intelligence in Education. Exceedly Press, 2019.

[32] R. Luckin, K. R. Koedinger, and J. Greer (eds.), Artificial Intelligence in Education: Building Technology Rich Learning Contexts that Work IOS Press, 2007.

\section{ABOUT THE AUTHORS}

Matthew N.0. Sadiku is a professor emeritus in the Department of Electrical and Computer Engineering at Prairie View A\&M University, Prairie View, Texas. He is the author of several books and papers. His areas of research interests include computational electromagnetics and computer networks. He is a fellow of IEEE.
Tolulope J. Ashaolu is a doctoral student at Prince of Songkla University. He is the author of several papers and a book. His research interests include functional foods and food microbiology.

Abayomi Ajayi-Majebi is a professor in the Department of Manufacturing Engineering at Central State University in Wilberforce, Ohio. In 2015 he was honored by the White House as a Champion of Change for his significant contributions to the engineering education of minority students. He is a senior member of both the Society of Manufacturing Engineers and the American Society for Quality.

Sarhan M. Musa is a professor in the Department of Electrical and Computer Engineering at Prairie View A\&M University, Prairie View, Texas. He has been the director of Prairie View Networking Academy, Texas, since 2004. He is an LTD Sprint and Boeing Welliver Fellow. His areas of research interests include computational electromagnetics and computer networks.

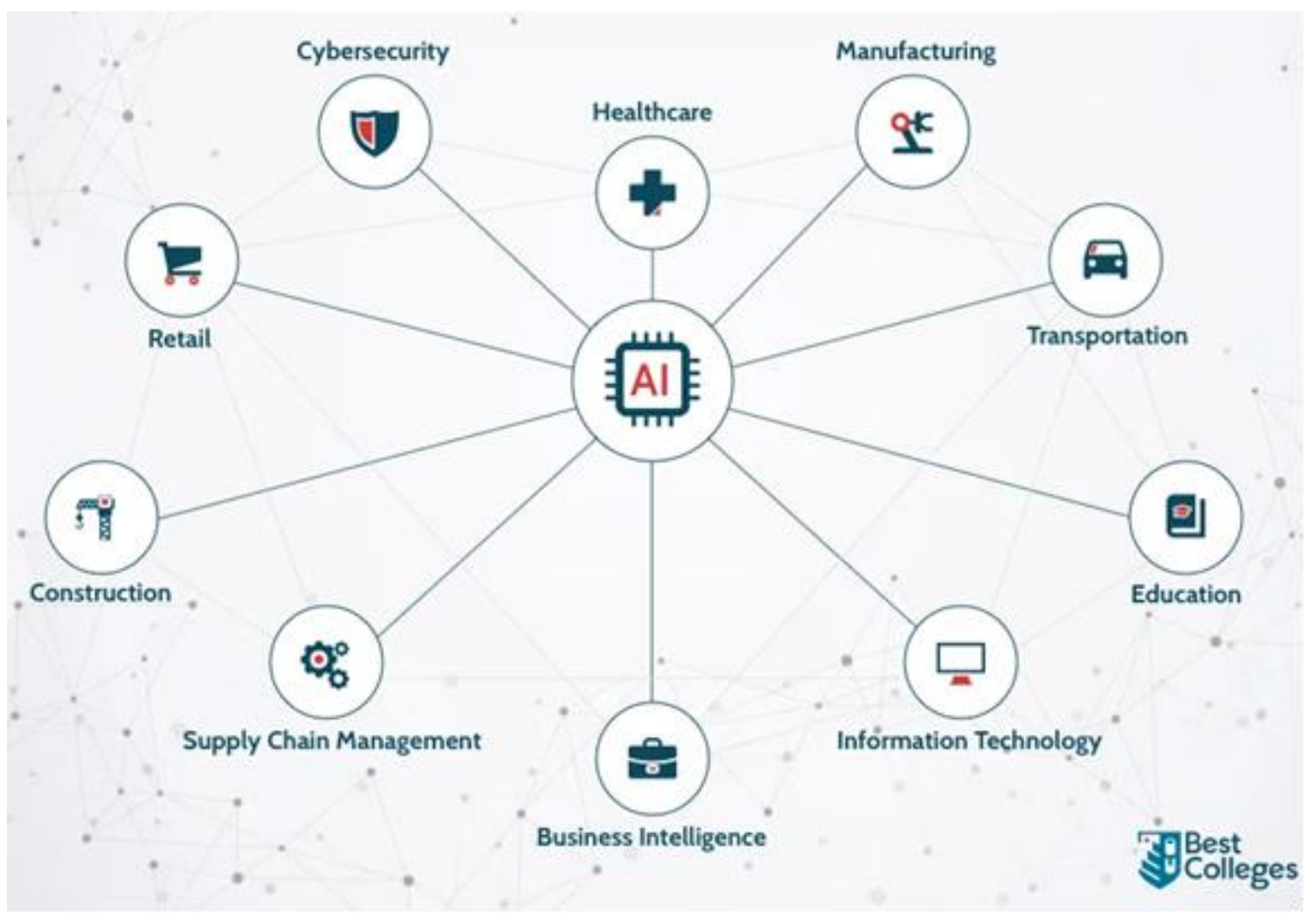

FIGURE 1: Industries impacted by AI revolution [2]. 


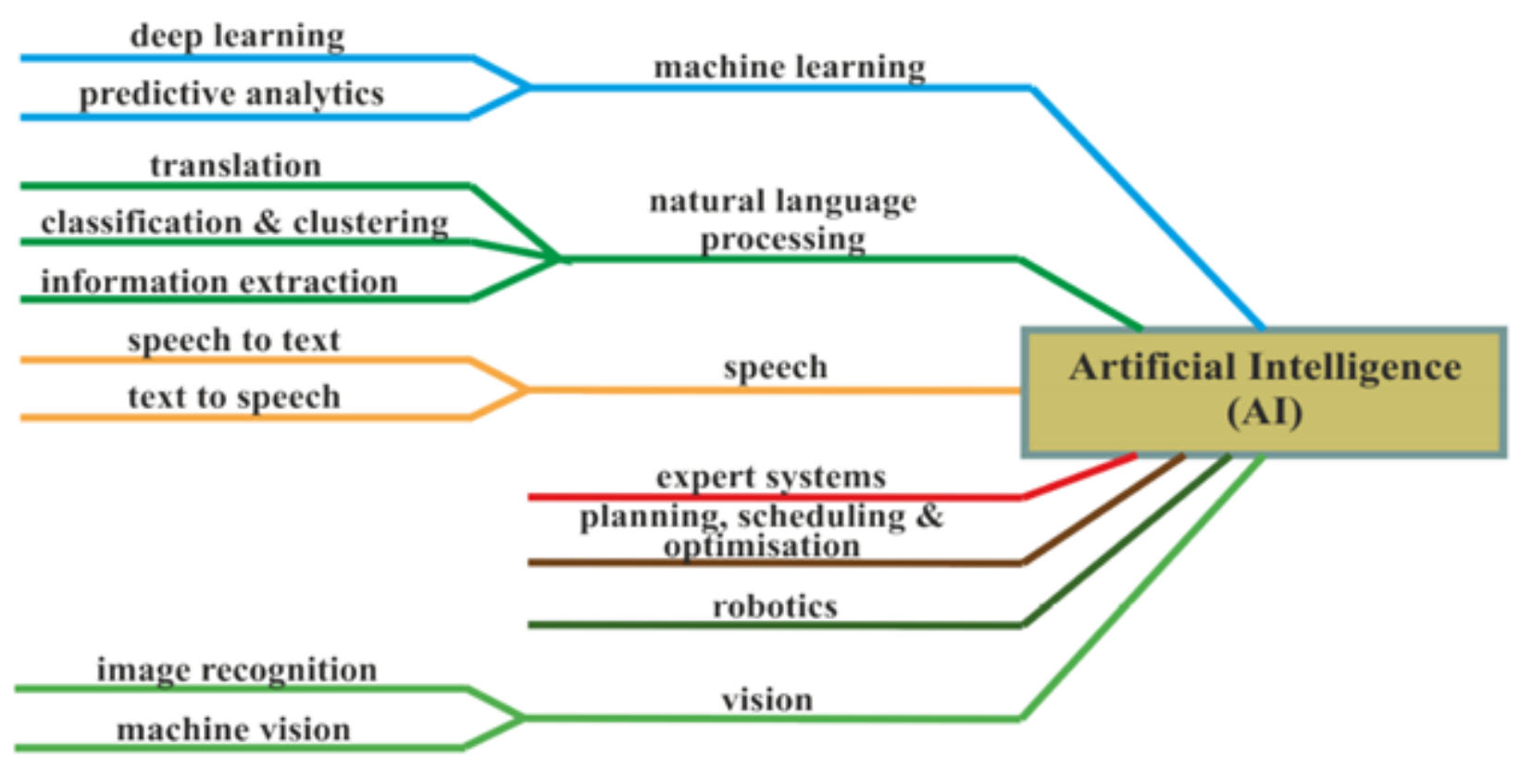

FIGURE 2: Many branches of artificial intelligence [7].

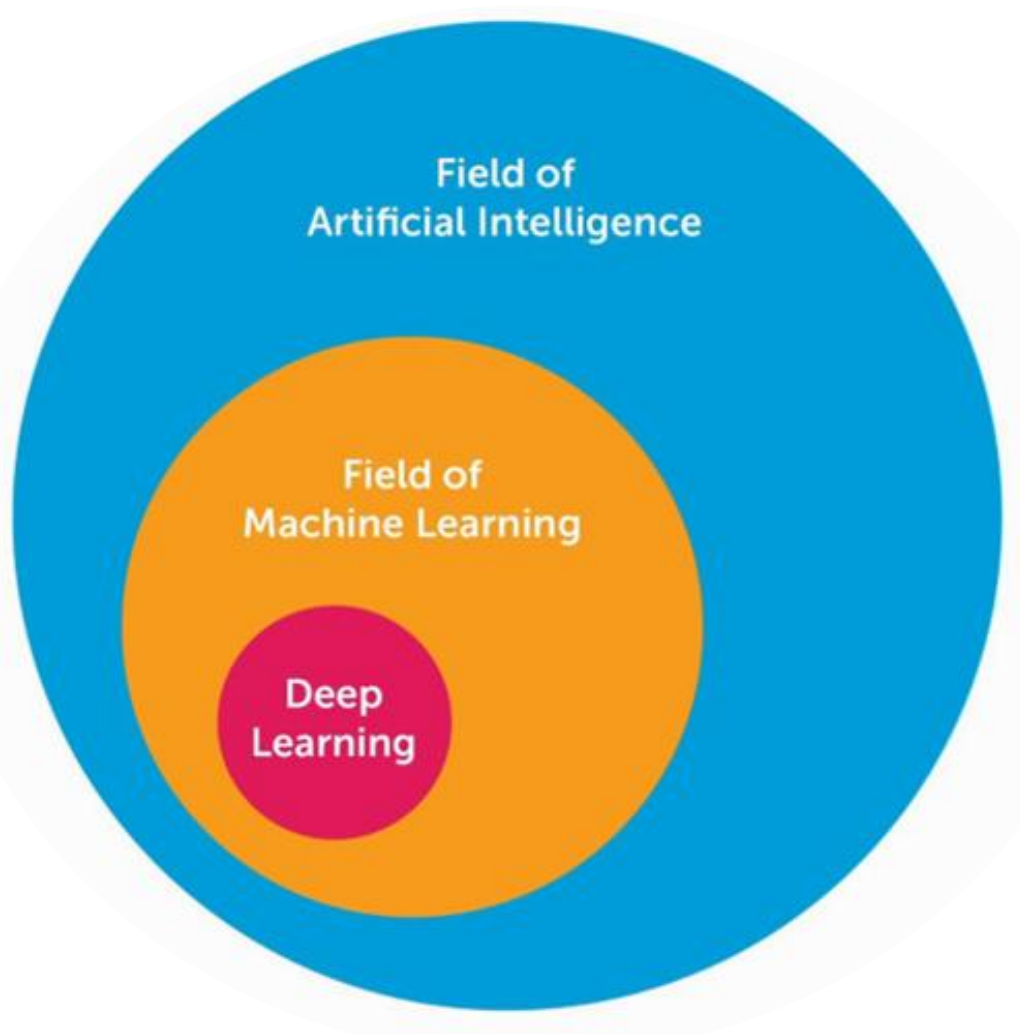

FIGURE 3: The relationship between AI, machine learning, and deep learning [13]. 


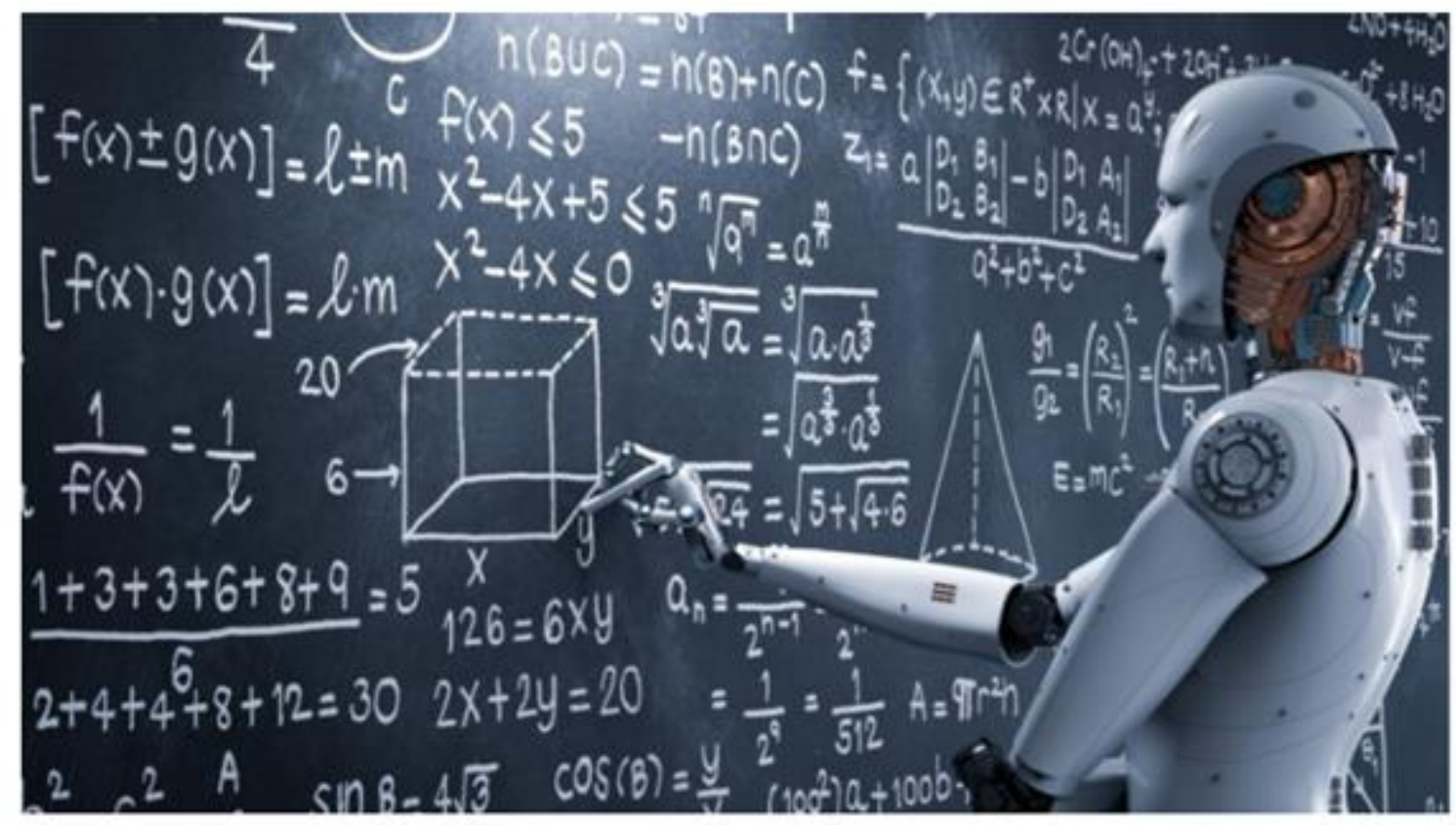

FIGURE 4: A typical example on how AI is changing the education industry [14].

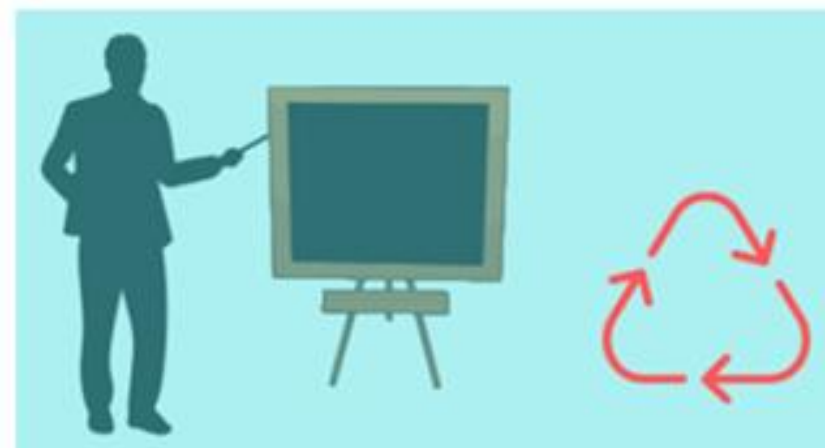

Traditional

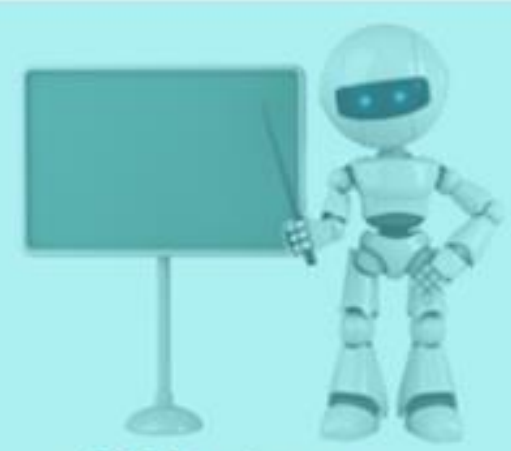

AI-Driven

FIGURE 5: Comparing traditional education with AI-driven education [17]. 